\title{
Customized 3D Bolus Applied to the Oral Cavity and Supraclavicular Area for Head and Neck Cancer
}

\author{
SEUNGHYEOP BAEK ${ }^{1,2}$, SOHYUN AHN ${ }^{1}$, EUNBIN JU ${ }^{1,3}$ and NURI HYUN JUNG ${ }^{1}$ \\ ${ }^{1}$ Department of Radiation Oncology, Kangwon National University Hospital, Kangwon, Republic of Korea; \\ ${ }^{2}$ Department of Radiological Science, School of Yonsei University, Wonju, Republic of Korea; \\ ${ }^{3}$ Department of Bio-medical Science, Graduate School of Korea University, Sejong, Republic of Korea
}

\begin{abstract}
Background/Aim: In this study, a new method to create a customized three-dimensional (3D) bolus by accurately considering the anatomy of an individual patient is demonstrated. Patients and Methods: A 3D bolus structure was created from an extended planning target volume (PTV) to reduce an inevitable skin reaction. In addition, during computed tomography simulation in patients with oral cavity cancers, a balloon was inserted into the mouth of a patient to secure space, and then the area surrounding the balloon was designed into a $3 D$ bolus structure. Results: For patients with head and neck cancers, a customized $3 D$ bolus can reduce the unnecessary skin dose by $14.4 \%$ compared to a commercial bolus. For patients with oral cavity cancer, the PTV and tongue doses were $93.8 \%$ and $8 \%$ of the prescribed dose, respectively. Conclusion: The customized $3 D$ bolus enables effective skin sparing and full coverage of the target area.
\end{abstract}

In addition to surgery and chemotherapy, radiation therapy (RT) is one of the key treatments of cancer. Considering the size, location, and extent of metastasis, the RT is used alone or in combination with other treatments. Although the purpose of RT is to control tumors, there is a possibility of adverse effects on normal tissues, which makes it a limiting factor when deciding the radiation dose.

Following RT, the side effects frequently experienced by patients with head and neck $(\mathrm{H} \& \mathrm{~N})$ cancers are lethargy and body weakness. In particular, if the treatment site is oral, side effects such as mucositis and sore throat owing to toxic doses of irradiation to the mucous membrane, dry mouth and oral

This article is freely accessible online.

Correspondence to: Sohyun Ahn, Ph.D., Professor of Kangwon National University Hospital, 24289, Kangwon, Republic of Korea. Tel: +82 332589390, Fax: +82 332589472, e-mail: mpsohyun@gmail.com

Key Words: Customized 3D bolus, head and neck cancer, skin reaction, radiation therapy, in vivo dosimetry, tongue immobilization. mucosa disease owing to tongue irradiation, loss of saliva, change in taste sensation, and others may occur. According to Rose-ped et al., the most common side effects of RT experienced by the patients with $\mathrm{H} \& \mathrm{~N}$ cancers are pharyngitis, oral mucosal disease, and dry mouth, which account for 20 , 18 , and $14 \%$ of the total number of side effects encountered, respectively. Furthermore, in the case where the treatment site was the oral cavity, approximately $90 \%$ of the patients experienced a change in taste function and $75 \%$ of them experienced a change in their oral health (1).

Another side effect of RT is skin reactions, mainly in the form of sunburn and can be classified into grades 1-4 depending on the severity of the damage (2). In the RTOG 90-03 study, toxicities according to the RT techniques were divided into grades 1-4 for locoregionally advanced H\&N cancer patients. Further, the symptoms corresponding to grades 1 and 2 were commonly observed among the patients; some patients exhibited the symptoms corresponding to grades 3 and 4 (3).

Fe et al. have evaluated the skin dose in intensitymodulated radiotherapy (IMRT) using a thermoluminescence dosimeter (TLD) and an EBT2 film (4). They observed skin reactions corresponding to grade 3 when the skin doses were higher than 40 Gy for 30 fractions.

Skin reactions in patients with $H \& N$ cancers is one of the more important side effects, and efforts to reduce them are currently being investigated in the medical field.

The limit of recoverable dose varies depending on the irradiated area. Zopate et al. have reported that the smaller the irradiation area the higher the recoverable dose to the skin, and the wider the irradiation area the lower the recoverable dose (5). This indicates that by narrowing the area of the skin to which the high dose is irradiated, the recoverable dose of the skin is increased, thereby reducing the probability of skin damage by radiation.

The use of bolus for superficial lesion treatment is a significantly useful method since it creates an appropriate dose buildup. However, when a commercial bolus is used on areas of the body with irregular skin, e.g., $\mathrm{H} \& \mathrm{~N}$, the bolus 


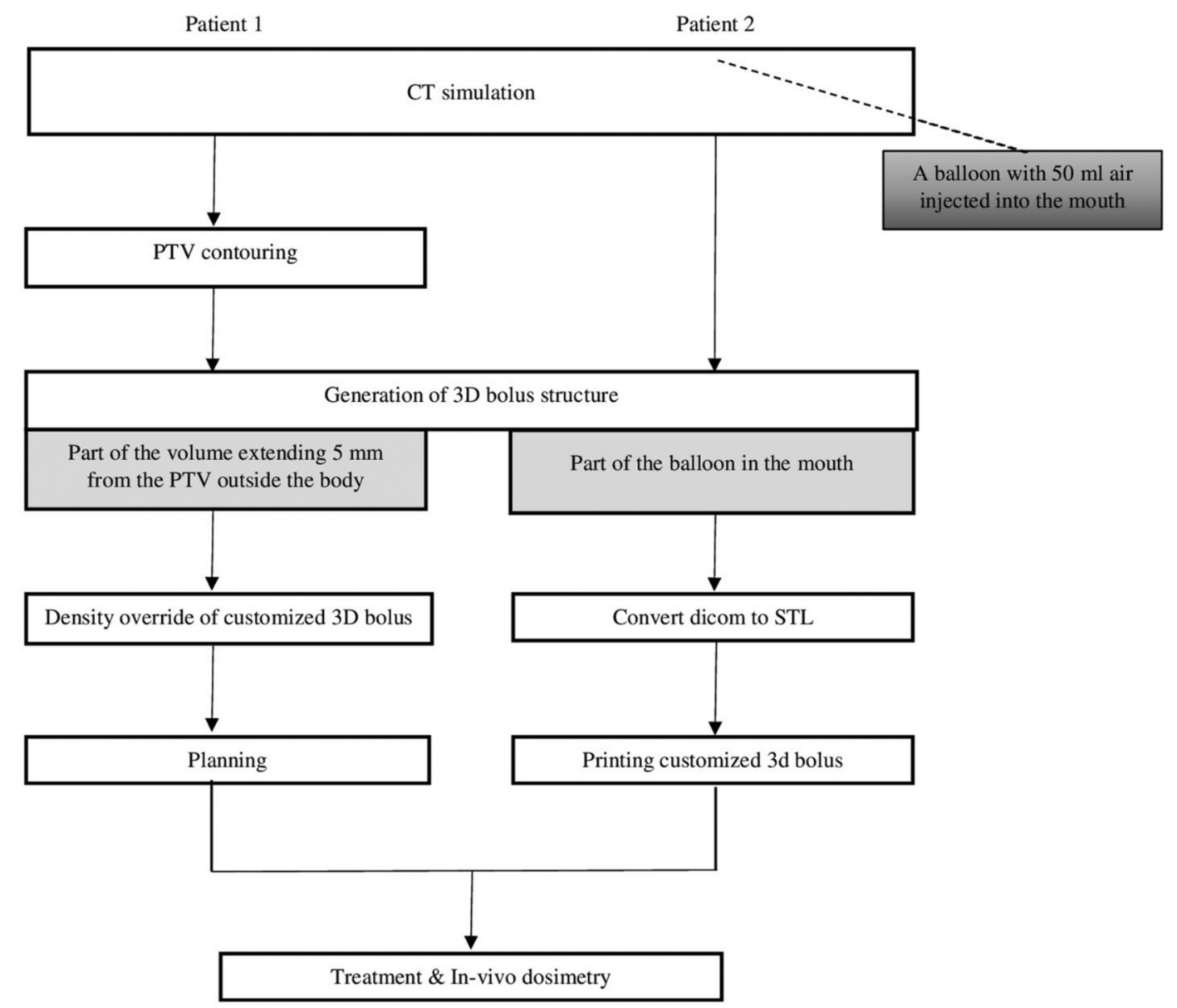

Figure 1. Workflow for manufacturing a customized $3 D$ bolus.

Table I. Basic and treatment information of patient 1 and patient 2.

\begin{tabular}{lcc}
\hline $\begin{array}{l}\text { Patient characteristic } \\
\text { and treatment }\end{array}$ & Patient 1 & Patient 2 \\
\hline $\begin{array}{l}\text { Gender } \\
\text { Age }\end{array}$ & Male & Female \\
Prescribed dose & $61 \mathrm{y}$ & $69 \mathrm{y}$ \\
Treatment site & $66 \mathrm{~Gy} / 30 \mathrm{fx}$ & $45 \mathrm{~Gy} / 10 \mathrm{fx}$ \\
& Larynx-bilateral & Oral cavity \\
neck & (Hard palate) \\
Treatment technique & 2 full arcs, 6 MV & 2 full arcs, 6 MV \\
Year of treatment & $04 / 2020$ & $05 / 2020$ \\
Purpose of RT & Curative treatment & Curative treatment \\
\hline
\end{tabular}

does not exactly match the skin, resulting in an air gap between the bolus and the skin. Owing to this air gap, the dose buildup does not occur effectively, for not only it does not irradiate the required dose to the specified treatment site, but it also increases the risk of damage due to excess doses reaching areas of the skin outside the treatment site.
Table II. Relative dose comparison with commercial and customized $3 D$ boluses.

\begin{tabular}{lc}
\hline & $\begin{array}{c}\text { Relative dose compared to 10-mm } \\
\text { commercial bolus [\%] (Measurement) }\end{array}$ \\
\hline w/o bolus & 20.7 \\
3 mm commercial bolus & 75.9 \\
5 mm commercial bolus & 96.1 \\
Customized 3D bolus & 88.5 \\
\hline
\end{tabular}

In addition, there is a problem of position reproducibility due to changes in the location of the air gap during repeated treatments. For helical tomotherapy (HT) and direct tomotherapy (DT), Akasaka et al. have shown through a focused plan study that the air gap between the bolus and the skin may vary with each treatment, and DT has a more serious air gap effect than HT (6). Therefore, there is a need for a new type of bolus that prevents the formation of an air 

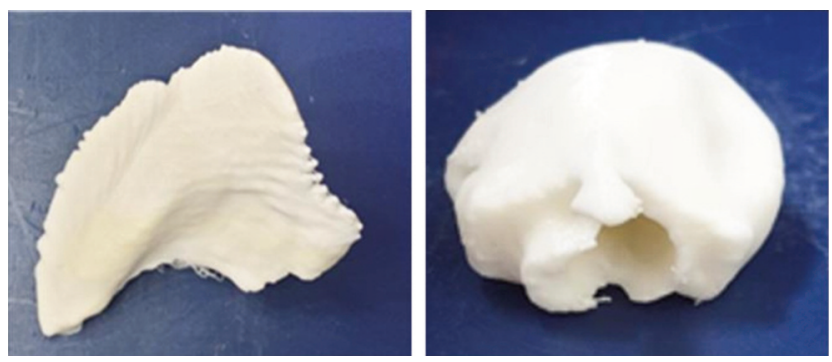

Figure 2. Customized 3D bolus for (a) patient 1 and (b) patient 2. The $3 D$ bolus in (a) is generated from the extended tumor in CT of patient 1 to provide a sufficient dose to the tumor located in the shallow area of the skin. (b) The 3D bolus of a $50 \mathrm{ml}$ balloon inserted into the mouth patient 2 during CT scan. An air cavity was created in the middle of the bolus for the patient to breathe comfortably.

Table III. Relative dose comparison of in vivo dosimetry for patient 1 .

\begin{tabular}{lcccc}
\hline Area & \multicolumn{4}{c}{ Relative dose compared to prescribed dose [\%] } \\
\cline { 2 - 5 } & $\begin{array}{c}\text { w/o bolus } \\
\text { (Plan) }\end{array}$ & $\begin{array}{c}0.5 \mathrm{~cm} \\
\text { bolus } \\
\text { (Plan) }\end{array}$ & $\begin{array}{c}\text { Customized } \\
\text { 3D bolus } \\
\text { (Plan) }\end{array}$ & $\begin{array}{c}\text { Customized } \\
\text { 3D bolus } \\
\text { (Measurement) }\end{array}$ \\
\hline $\begin{array}{l}\text { Skin sparing area } \\
\text { Tumor area }\end{array}$ & 34.8 & 73.0 & 41.6 & 58.6 \\
\hline
\end{tabular}

gap, i.e., a customized patient-specific bolus designed to match the irregular surface of a specific patient.

The 3D printing technology is employed in various medical fields, e.g., for making artificial bones or organs (7, 8 ), and it has also been used in several institutions in the field of RT. Furthermore, Asfia et al. have made immobilizers using 3D printers to minimize patient movement, and reviewed various studies that have used these immobilizers (9).

Park et al. applied a 3D bolus, produced from 3D printing, on a breast-one of the irregular skin areas in the human body-along with the H\&N. Further, the calculated dose distribution and the measured dose were compared with the commercial bolus. They have reported that the patientspecific 3D bolus can reduce the air gap between the skin and the bolus, thereby reducing the inaccuracies caused by changing daily clinical setups (10).

Canters et al. have reported that applying a 3D printed bolus in electron beam treatment can increase target coverage compared to a commercial bolus (11). Chiu et al. have reported that a patient-specific 3D bolus can be applied clinically, even when considering the efficiency of time and cost (12).

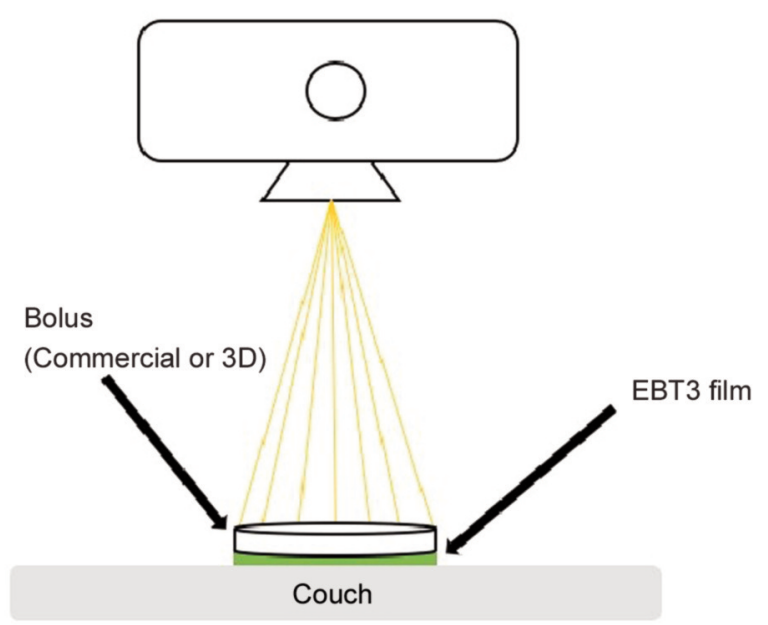

Figure 3. Experiment to compare the dose buildup effect of a $3 D$ bolus and a commercial bolus in standard conditions. Standard condition: 6 $M V X$-ray beam with a field size and source-to-surface distance (SSD) of $10 \times 10 \mathrm{~cm}^{2}$ and $100 \mathrm{~cm}$, respectively. The doses of 3-, 5-, and 10-mm thick commercial boluses and the customized $3 D$ bolus were compared under standard conditions. An EBT3 film was used for the dose measurement

In this study, we propose a new method of producing a customized 3D bolus, with the purpose of improving the target coverage and positioning reproducibility.

\section{Patients and Methods}

Patients. The patient's dose was evaluated in two patients with H\&N cancers. Each of the two patients were treated with a customized 3D bolus. Furthermore, these two patients were selected from the patients who received RT at Kangwon national University Hospital in 2020 (Table I). Patients 1 and 2 received curative treatment with $66 \mathrm{~Gy} / 30 \mathrm{fx}$ and $45 \mathrm{~Gy} / 10 \mathrm{fx}$ for the larynx-bilateral necks and oral cavity (hard palate), respectively. Both the patients were treated with VMAT (2 full arcs, $6 \mathrm{MV}$ ) using a linear accelerator (Clinac iX, Varian Medical Systems, Palo Alto, CA, USA).

Manufacturing workflow for a customized 3D bolus. Figure 1 shows the workflow of manufacturing a customized 3D bolus. Since X-ray has a characteristic that the dose is delivered after passing through a buildup section of several millimeters, it must be located at least $5 \mathrm{~mm}$ inside the skin to sufficiently deliver the prescribed dose to the tumor. However, if the tumor is located very close to the skin, a bolus that can serve as a buildup must be produced.

For patient 1, after the radiation oncologist contoured the gross tumor volume and planning target volume (PTV) in the computed tomography (CT) image, the PTV was uniformly extended by $5 \mathrm{~mm}$ to allow the application of the customized 3D bolus only to the part that satisfies the target coverage, when dose buildup was performed using a bolus located close to the surface. Among the extended PTVs, the area outside the body, i.e., the area where the PTV is close to the skin surface, was set as a customized 3D bolus structure. 

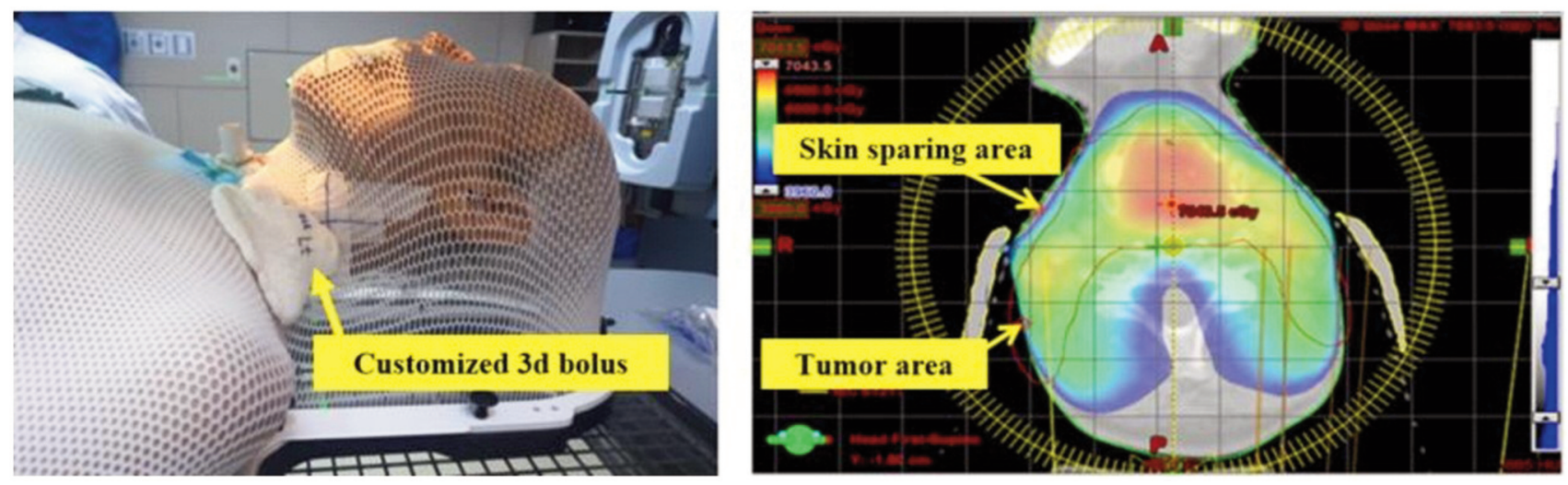

Figure 4. Treatment setup and dose distribution in the treatment planning for patient 1. (a) Patient 1 is an H\&N cancer patient. A customized 3D bolus was created for dose buildup in the area where the tumor was adjacent to the skin and attached to the mask for immobilization during the treatment. Since the customized 3D bolus was created based on the patient's CT, it fits well with the patient's body. The same area viewed as an axial image in the treatment plan is shown in (b). The region below the customized $3 D$ bolus is the buildup area in the axial image, and the area without the bolus is the skin sparing area.

Table IV. Relative dose comparison of in vivo dosimetry for patient 2.

\begin{tabular}{lcc}
\hline Area & \multicolumn{2}{c}{ Relative dose compared to prescribed dose [\%] } \\
\cline { 2 - 3 } & $\begin{array}{c}\text { Customized } \\
\text { 3D bolus } \\
\text { (Plan) }\end{array}$ & $\begin{array}{c}\text { Customized } \\
\text { 3D bolus } \\
\text { (Measurement) }\end{array}$ \\
\hline PTV & 92.4 & 93.8 \\
Tongue & 22.5 & 8.0 \\
\hline
\end{tabular}

For patient 2, when performing a CT simulation, a balloon was inserted into the patient's mouth, $50 \mathrm{ml}$ of air was injected in the balloon to fix the position of the tongue, and an image was acquired. In the CT image, the volume of the balloon was defined as a customized $3 \mathrm{D}$ bolus.

During the treatment planning, the dose was calculated by overriding the density of the bolus to the density of polylactic acid, which was $1.24 \mathrm{~g} / \mathrm{cm}^{3}$. The generated 3D bolus structure was saved in a dicom file format and converted into Standard Triangle Language (STL) file to create a customized 3D bolus using a 3D printer (3DWOX eco, Sindoh, Seoul, South Korea).

The customized 3D boluses for patients 1 and 2 are shown in Figure 2.

Dose evaluation. In vivo dosimetry was performed to verify the dose coverage of tumor lesions and spare effects on normal tissue. In the case of patient 1 , to verify the sufficient dose buildup in the skin near the tumor due to the customized 3D bolus, an EBT3 film for dose measurement was placed under the customized 3D bolus. Further, the performance of skin sparing for dose in the skin irrelevant to tumor was evaluated. In addition, to evaluate the buildup effect on the customized 3D bolus produced for patient 1 , compared to the commercial bolus, the dose was evaluated under the same conditions $\left(6 \mathrm{MV}, 100 \mathrm{MU}, 10 \times 10 \mathrm{~cm}^{2}\right.$ field size, SSD

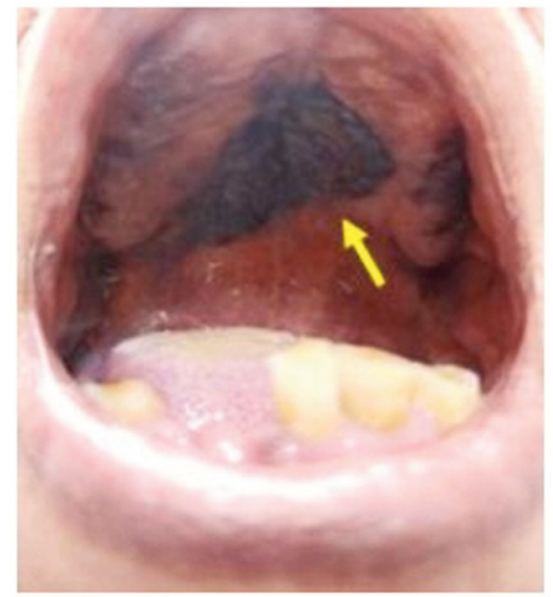

Figure 5. Treatment site of patient. The tumor lesion of patient 2 is located in the hard palate area; hence, dose buildup is required. Simultaneously, the tongue can be fixed as far as possible from the lesion to reduce unnecessary dose exposure.

$100 \mathrm{~cm}$ ) as the commercial bolus with 3,5 , and $10 \mathrm{~mm}$ thicknesses. The experimental setup is shown in Figure 3. In the case of patient 2 , the target coverage was evaluated between the hard palate and the customized $3 \mathrm{D}$ bolus, which is the treatment site, and the dose was also evaluated between the tongue and the customized 3D bolus.

\section{Results}

Patient 1. The comparison of the dose between the commercial bolus and the customized 3D bolus of patient 1 showed that the customized 3D bolus was equivalent in terms of the dose to the commercial bolus of approximately $4.3 \mathrm{~mm}$ thickness, 

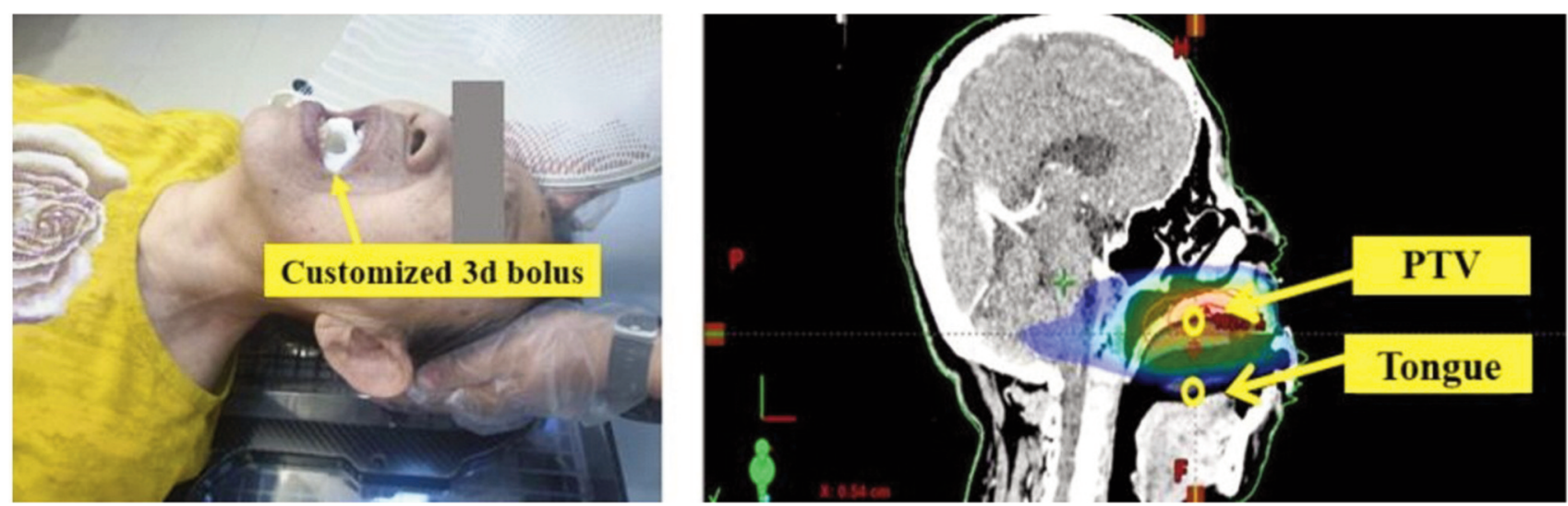

Figure 6. Treatment setup and planning for patient 2. (a) Treatment setup and applying the customized 3D bolus made to patient 2, (b) a sagittal CT image taken with a balloon injected with $50 \mathrm{ml}$ air in the mouth.

and it allowed approximately $7.6 \%$ lower dose than that of the 5-mm thick bolus (Table II). Figure 4a illustrates how the customized 3D bolus was applied during the treatment of patient 1 , and Figure $4 \mathrm{~b}$ shows the axial dose distribution of the dose-calculated plan including the density override 3D bolus. In Figure $4 b$, the skin sparing area is the area where the tumor is located deep from the skin; hence, the skin does not receive a large dose, equivalent to the prescribed dose. In contrast, the tumor area is the area where the PTV is located close to the skin and requires dose buildup, and the area where the bolus structure is created from the extended PTV.

The dose distributions at these two points are shown in Table III. The doses at the skin sparing area were $34.8 \%$ and $73.0 \%$ without and with commercial bolus, respectively. Further, when a customized 3D bolus was applied at the skin sparing area, the dose value was $41.6 \%$, which is similar to the dose value without a bolus.

For the tumor area, the dose was $41.3 \%$ without any bolus, $82.7 \%$ for the 5 -mm thick commercial bolus, and $67.5 \%$ for the customized 3D bolus. When patient 1 treated with the customized $3 \mathrm{D}$ bolus, the in vivo measurements showed $58.6 \%$ and $83.2 \%$ of the prescribed dose at skin sparing area and tumor area, respectively.

Patient 2. The treatment site of patient 2 is shown in Figure 5. Moreover, the treatment setup and the axial dose distribution of the dose-calculated plan, including density override 3D bolus, is shown in Figure 6a and b, respectively. In Figure 6b, the PTV region is the area that requires dose buildup and the tongue region is the area where the dose buildup is not required. The treatment plan dose and the measured dose in the tumor area of the hard palate were $92.4 \%$ and $93.8 \%$, respectively. The dose to the tongue in the treatment plan and in vivo measurement were $22.5 \%$ and $8.0 \%$, respectively (Table IV).

\section{Discussion}

In RT, improving patient outcomes while reducing side effects is always a major concern. All medical staff involved in the patient's treatment discussed together the proper treatment and application of a customized 3D bolus, and performed in vivo dosimetry. As a result, the patient-specific treatment was well progressed.

In the case of patient 1 , unlike producing the $3 \mathrm{D}$ boluses using previously reported methods, the benefit of the production of $3 \mathrm{D}$ boluses by expanding the PTV, which is the method proposed in this study, is that they can be made for specific areas of the human body. This method can be applied in the future to other sites such as breasts, hands, and feet.

As a result of the dose measurement for patient 1, if a 5$\mathrm{mm}$ thick bolus was used for the entire area, it can be expected that $48.2 \mathrm{~Gy} / 30 \mathrm{fx}$ would be irradiated in the area where the dose buildup was unnecessary (Figure 4). According to $\mathrm{Fu}$ et al. (3), the skin reactions corresponding to grade 2 will occur in the skin exposed to this dose. Alternatively, owing to the use of the $3 \mathrm{D}$ bolus only in the local area, the dose to the area where the dose buildup is not required is reduced to $24.9 \mathrm{~Gy} / 30 \mathrm{fx}$, and a skin reaction corresponding to grade 1 or less is expected. Therefore, it is obvious that the method used in this study is useful for skin sparing.

In the case of patient 2, applying a 3D bolus to the oral cavity not only produces a better buildup effect, but also has the advantage of immobilizing the tongue; hence, the patient may feel less discomfort during the IMRT treatment. In addition, a hole in the middle of the bolus was created to allow the patient to breathe comfortably, and the shape of the 3D bolus was carefully made to match the mouthpiece worn by the patient and to improve reproducibility during daily repetitive treatment. The $3 \mathrm{D}$ bolus production is difficult to 
apply to many patients because it requires large production time and work force. However, through this study, the possibility of the customized treatment was confirmed through the cooperation among the members of the department.

\section{Conclusion}

In this study, we found that creating a local 3D bolus structure using an extended PTV can improve target coverage and reduce unnecessary skin dose for an irregular skin. In addition, when an oral cavity is treated, the space created in advance using a balloon in the CT simulation step can be made into a 3D bolus structure, so that even when the patients are treated with the $3 \mathrm{D}$ bolus inserted in the oral cavity, they can be treated naturally and comfortably. This not only improves target coverage, but also achieves a tongue immobilization effect.

\section{Conflicts of Interest}

The Authors certify that they have no affiliations with or involvement in any organization or entity with any financial interest (such as honoraria; educational grants; participation in speakers' bureaus; membership, employment, consultancies, stock ownership, or other equity interest; and expert testimony or patent-licensing arrangements), or non-financial interest (such as personal or professional relationships, affiliations, knowledge or beliefs) in the subject matter or materials discussed in this manuscript.

\section{Authors' Contributions}

All Authors certify that they have participated sufficiently in the work to take public responsibility for the content, including participation in the concept, design, analysis, writing, or revision of the manuscript. Furthermore, each Author certifies that this material or a similar material has not been submitted to or published in any other journal.

\section{Acknowledgements}

This work was supported by the National Research Foundation of Korea (NRF) grant funded by the Korea government (MSIT) (No. 2019R1A2C108912912).

\section{References}

1 Rose-Ped AM, Bellm LA, Epstein JB, Trotti A, Gwede C and Fuchs HJ: Complications of radiation therapy for head and neck cancers. Cancer Nurs 25(6): 461-467, 2002. PMID: 12464838. DOI: $10.1097 / 00002820-200212000-00010$

2 Cox JD, Stetz J and Pajak TF: Toxicity criteria of the radiation therapy oncology group and the european organization for research and treatment of cancer. Int J Radiat Oncol Biol Phys 31(5): 1341-1346, 1995. PMID: 7713792. DOI: 10.1016/03603016(95)00060-C
3 Fu KK, Pajak TF, Trotti A, Jones CU, Spencer SA, Phillips TL, Garden AS, Ridge JA, Cooper JS and Ang KK: A Radiation Therapy Oncology Group (RTOG) phase III randomized study to compare hyperfractionation and two variants of accelerated fractionation to standard fractionation radiotherapy for head and neck squamous cell carcinomas: first report of RTOG 9003. Int J Radiat Oncol Biol Phys 48(1): 7-16, 2000. PMID: 10924966. DOI: $10.1016 / \mathrm{s} 0360-3016(00) 00663-5$

4 Fu HJ, Li CW, Tsai WT, Chang CC and Tsang YW: Skin dose for head and neck cancer patients treated with intensitymodulated radiation therapy (IMRT). Radiat Phys Chem 140: 435-441, 2017. DOI: 10.1016/j.radphyschem.2017.02.017

5 Waghmare CM: Radiation burn-From mechanism to management. Burns 39(2): 212-219, 2013. PMID: 23092699. DOI: $10.1016 /$ j.burns.2012.09.012

6 Akasaka H, Oki Y, Mizonobe K, Uehara K, Mayahara H, Harada A, Hashimoto N, Kitatani K, Yabuuchi T, Ishihara T, Iwashita K, Miyawaki D, Mukumoto N, Nakaoka A and Sasaki R: The air gap between bolus and skin affects dose distribution in helical and direct tomotherapy. J Radiother Pract 1-6, 2020. DOI: $10.1017 /$ S1460396920000333

7 Popov Jr, Muller-Kamskii G, Kovalevsky A, Dzhenzhera G, Strokin E, Kolomiets A and Ramon J: Design and 3D-printing of titanium bone implants: brief review of approach and clinical cases. Biomed Eng Lett 8(4): 337-344, 2018. PMID: 30603218. DOI: $10.1007 / \mathrm{s} 13534-018-0080-5$

8 Radenkovic D, Solouk A and Seifalian A: Personalized development of human organs using 3D printing technology. Med Hypotheses 87: 30-33, 2016. PMID: 26826637. DOI: 10.1016/j.mehy.2015.12.017

9 Asfia A, Novak JI, Mohammed MI, Rolfe B and Kron T: A review of $3 \mathrm{D}$ printed patient specific immobilisation devices in radiotherapy. Phys Imaging Radiat Oncol 13: 30-35, 2020. DOI: 10.1016/j.phro.2020.03.003

10 Park SY, Choi CH, Park JM, Chun MS, Han JH and Kim JI: A patient-specific polylactic acid bolus mad by a $3 \mathrm{D}$ printer for breast cancer radiation therapy. PLoS One 11(12): e0168063, 2016. PMID: 27930717. DOI: 10.1371/journal.pone.0168063

11 Canters RA, Lips IM, Wendling M, Kusters M, Zeeland MV, Gerritsen RM, Poortmans P and Verhoef CG: Clinical implementation of $3 \mathrm{D}$ printing in the construction of patient specific bolus for electron beam radiotherapy for non-melanoma skin cancer. Radiother Oncol 121(1): 148-153, 2016. PMID: 27475278. DOI: 10.1016/j.radonc.2016.07.011

12 Chiu T, Tan J, Brenner M, Gu X, Yang M, Westover K, Strom T, Sher D, Jiang S and Zhao B: Three-dimensional printer-aided casting of soft, custom silicone boluses (SCSBS) for head and neck radiotherapy. Pract Radiat Oncol 8(3): e167-e174, 2018. PMID: 29452869. DOI: 10.1016/j.prro.2017.11.001

Received October 21, 2020

Revised November 9, 2020

Accepted November 10, 2020 